\title{
Estado físico-químico del suelo en pasturas con y sin maní forrajero (Arachis pintoi), URACCAN-Siuna
}

\author{
Jeffer Márquez Chow' \\ José Ángel Rodríguez Morales ${ }^{2}$ \\ Manuel Marín Castellón ${ }^{3}$
}

\section{Resumen}

$\mathrm{C}^{1}$ estudio fue desarrollado en un área de una manzana, propiedad del señor Marvin Calderón, localizada en la comunidad Yaoya, municipio de Siuna. El objetivo fue comparar el estado físico-químico del suelo en pasturas con y sin maní forrajero (Arachis pintoi). Es cuasi experimental pre test, post test, longitudinal y descriptivo y se utilizó la técnica observacional. Se concluye, que el Arachis pintoi es una especie que contribuye al mejoramiento de la calidad del suelo y se recomienda el uso de prácticas agronómicas antes y después de establecer el mismo. El establecimiento del Arachis pintoi y la parcela de pasto sin este, cuestan en total $C \$ 7,377$. oo.

Palabras clave: suelo, mejoramiento, nutrientes, nitrógeno, estructura, textura.

\section{Summary}

The study was developed in an area of one block, owned by Mr. Marvin Calderón, located in Yaoya Community, municipality of Siuna. The objective was to compare the physical-chemical state of the soil in pastures with and without fodder peanut (Arachis pintoi). It is quasi-experimental pre-test, post-test, longitudinal and descriptive study and the observational technique was used. It is concluded that the Arachis pintoi is a species that contributes to the improvement of the soil quality and it is recommended the use of agronomic practices before and after establishing the same. The establishment of Arachis pintoi and the grass plot without it cost $C \$ 7,377.00$ in total.

Key Words: soil, improvement, nutrients, nitrogen, structure, texture.

\section{Introducción}

Uno de los grandes retos de la humanidad es la racionalización en el uso y explotación de los recursos naturales, dentro de los cuales se encuentra el recurso suelo, como un sistema vivo y sinérgico. El suelo es un medio de producción agrícola importante, desde el cual las plantas suplen sus requerimientos hídricos y nutricionales. Es considerado como un recurso natural renovable: sin embargo, esta capacidad de renovación depende en gran medida de los usos y manejo que este recurso haya recibido (Silva, 2008).

Nicaragua ha sido un país con una larga trayectoria agrícola y ganadera, fundamentada en las excelentes características de los suelos; abundantes recursos hídricos superficiales y subterráneos para la irrigación; condiciones climáticas favorables, aunque afectadas en algunas zonas por sequías inter estacionales y un

\footnotetext{
1 Ingeniero Zootecnista por la Universidad de las Regiones Autónomas de la Costa Caribe Nicaragüense-Recinto Universitario Las Minas. Correo: Jeffermarquez92@gmail.com

2 Ingeniero Zootecnista por la Universidad de las Regiones Autónomas de la Costa Caribe Nicaragüense-Recinto Universitario Las Minas. Correo: jsurditomorales@gmail.com

3 Máster e Ingeniero de Minas. Profesor de la Universidad de las Regiones Autónomas de la Costa Caribe Nicaragüense-Recinto Universitario Las Minas. Correo: mannymarinc12@yahoo.es
}

Recibido: 06/10/2017 Aprobado: 23/11/2017 
potencial humano con alta tradición agropecuaria (Budhu, 2007).

En Nicaragua, el recurso suelo es uno de los más deteriorados por el mal uso, con prácticas agrícolas inadecuadas y no reguladas, lo cual afecta los relieves que son sensibles a las fuerzas del viento y el agua. Aunque se conoce la importancia de los suelos en las actividades agrícolas, pecuarias y forestales, muy poco se hace para el manejo y la conservación. Según el Informe del Estado del Ambiente "GEO 2003", (Franklin, et al, 2007), uno de los problemas ambientales más importantes que enfrenta Nicaragua es el deterioro de la fertilidad de los suelos por razones de cambios de uso de suelos forestales para la agricultura y ganadería, incluyendo uso de laderas.

En la región y nuestro municipio, se destaca por ser una zona ganadera donde se les da un mal uso a los suelos, por el sobrepastoreo del ganado, este problema surge debido a la falta de utilización de técnicas y el manejo adecuado de las mismas para una mejor conservación de la fertilidad y la calidad del suelo.

Es por ello que a través del estudio, proponemos establecer una nueva técnica, que consiste en la combinación de (Arachis pintoi) como leguminosa con pasto, donde (Bourrillon, 2007), afirma que mejora las condiciones físicas y químicas del suelo por su aporte en nitrógeno, lo cual también contribuye a minimizar las pérdidas de nutrimentos por erosión, lixiviación y así también mejora la actividad biológica del mismo, y comparar con la práctica tradicional que consiste en el establecimiento de pastos sólo para el pastoreo.

\section{Revisión de literatura}

El suelo, un cuerpo natural que se encuentra en la superficie de la tierra con propiedades distintivas y proviene de distintos procesos: físicos, químicos y biológicos, que actuaron sobre el material madre imprimiéndole rasgos característicos que lo hacen capaz de soportar la vida vegetal (Pérez, 2009, p. 42).

La fertilidad del suelo es la capacidad de éste para mantener una cubierta vegetal. En la fertilidad intervienen todas las características del suelo, sean físicas, físico - químicas o químicas. Por ello se habla de una fertilidad asociada a cada una de ellas, si bien sólo serían aspectos parciales de un mismo concepto unitario (Mendoza, 2010).

La densidad aparente de un suelo es la relación que existe entre la masa o peso seco del suelo y la unidad de volumen aparente del mismo. El volumen aparente incluye a las partículas sólidas y el espacio poroso.

Suelos ácidos, son aquellos cuyo su potencial de hidrógeno es bajo, encontrándose en los rangos 3.5 a 5.5, en las cuales hay menor disponibilidad de nutrientes esenciales para las plantas (Zapata, 2006).

\section{Efectos del maní forrajero (Arachis pintoi) sobre las características físicas del suelo}

Se reconoce que la presencia de leguminosa en una asociación, favorece la estructura del suelo, minimizando las pérdidas de nutrientes por erosión y lixiviación y también favorece la actividad biológica.

Según Sampat (1991), las condiciones físicas del suelo se pueden modificar por su manejo y pueden alterarse radicalmente. Por eso es necesario que el manejo que se brinda al suelo, deba tener previamente un conocimiento claro de las condiciones físicas del mismo.

El maní forrajero perenne (Arachis pintoi), se ha convertido en una opción forrajera para mejorar los sistemas ganaderos y promover sistemas menos vulnerables y dependientes de ingredientes importados. Debido a su alta capacidad de fijación de nitrógeno, rápida degradación de sus hojarascas, estimulo sobre la diversidad biológica del suelo y mejoría en el contenido de materia orgánica en este, su presencia permite la recuperación de suelos degradados, lo que facilita su utilización como estrategia para mejorar la calidad de este recurso (Sampat, 1991, p. 6).

El estudio demostró que en las condiciones en las que se desarrolló el experimento (Arachis pintoi), alcanzó la mayor profundidad radicular $(36.5 \mathrm{~cm})$, superando significativamente a las profundidades alcanzadas por las demás coberturas. Seleccionar y manejar esta leguminosa como cultivo de cobertura que produce raíces profundas y mayores cantidades de biomasa radicular, son características deseables para incrementar la materia orgánica del suelo, mejorar la estructura del suelo, aumentar la capacidad retentiva de humedad del suelo y me- 
jorar la infiltración. La significancia funcional de la profundidad radicular y su contribución a todos los procesos del ecosistema todavía no están bien entendidos (Puertas y Arévalo, 2008, p. 22).

\section{Efectos del maní forrajero perenne sobre el estado químico del suelo}

Es conocida la particularidad que poseen la mayoría de leguminosas, de fijar el nitrógeno atmosférico al suelo, actuando de esta manera como mejoradoras de la fertilidad del mismo. Esto se debe a la simbiosis con microorganismos bacterianos del género Rizobium, los cuales viven saprofiticamente en el suelo, utilizando fuentes de energía y sustancias nitrogenadas del medio. Al infectar o ser inoculados a las raíces forman conglomerados celulares llamados nódulos, en los cuales es fijado el nitrógeno, facilitándose su absorción por la planta y a su vez, al envejecer la raíz o morir estos nódulos son aportados al suelo siendo aprovechados por otras plantas, entre ellas las gramíneas. Se refieren las cantidades de nitrógeno fijado por algunas plantas leguminosas entre 20 a $560 \mathrm{Kg}$. / ha año, dependiendo del suelo y de la humedad lo cual significa una economía nada despreciable en la inversión de estas.

\section{Recuperación de suelos degradados}

El retorno de nutrientes al suelo vía hojarasca producida por la planta, es generalmente de mayor importancia cuantitativa. El maní forrajero tiene un alto contenido de nutrientes con una alta incorporación al suelo debido a su rápida descomposición favoreciendo el reciclaje de nutrientes (Rincón, 1999).

Rincón (1999), demuestra que en pasturas asociadas por 3 y 9 años de (Arachis pintoi) y Brachiaria spp, cuantifica en mejoras sustanciales en el contenido de calcio, magnesio y materia orgánica.

\section{Importancia de las leguminosas en la agricultura}

El principal problema a enfrentar por los suelos para su utilización en la agricultura y en la producción de pastos, es su susceptibilidad a la degradación (Gómez, 2015, p. 20). En este sentido, es importante resaltar la importancia de proteger los suelos de los procesos de degradación, conceptualizada como la pérdida de algunas cualidades físicas, químicas y biológicas del suelo, por la inadecuada intervención humana, los cuales se convierten en factores negativos de producción y en el futuro afectarán la sostenibilidad agrícola (Gómez, 2015, p.20).

Es por ello, que el manejo y las labores de preparación de suelos deben enfocarse hacia la conservación y creación de una "capa arable" con una productividad progresiva. Se entiende en este contexto como capa arable a aquella capa superficial de suelo planificada y obtenida por el hombre, con el fin de obtener un suelo que no presente limitantes físicas, químicas ni biológicas para el desarrollo normal de las raíces de los cultivos y que sea estable a través del tiempo (Gómez, 2015, p.20).

Las plantas de cobertura de leguminosas, juegan un papel importante en la protección del suelo e incorporan cantidades apreciables de residuos que son rápidamente descompuestos, en función de su relación favorable de Carbono/Nitrógeno. El uso de especies leguminosas en la producción agropecuaria es una alternativa a considerar para el mantenimiento de la fertilidad del suelo. Estas especies protegen el suelo contra la erosión, aportan a éste materia orgánica (MO), nutrientes y mejoran sus propiedades; además, favorecen la retención de agua, la actividad biológica y reducen el uso de fertilizantes (Tecnologías y prácticas para pequeños productores agrarios,TECA, 2015).

Cuando los cultivos de cobertura se incorporan al suelo, la materia orgánica que se adiciona, se denomina abono verde. Cuando estos cultivos son sembrados directamente en asociación con otros cultivos, se denomina cobertura viva.

\section{Importancia y beneficios económicos del asocio de gramíneas con leguminosas}

Para un buen establecimiento y manejo de pastos y forrajes, con el fin de suministrar al ganado un alimento más abundante y de buena calidad, se debe considerar varios aspectos:

- Analizar el suelo donde va a establecer el pasto para determinar las necesidades de fertilizantes. Las gramíneas son exigentes especialmente en nitrógeno y fósforo y las leguminosas en calcio y fósforo.

- Preparar bien el suelo y disponer de un buen sistema de drenaje.

- Aplicar los correctivos al momento de la siembra, de acuerdo con el análisis del suelo y las recomendaciones. 
La duración y el rendimiento de los pastos dependen de la planificación del cultivo: época de siembra, fertilización, tanto en la siembra como en mantenimiento, deshierbas, riegos oportunos, momento adecuado de corte y/o pastoreo y resiembras.

\section{¿Qué son pastos asociados?}

Es la asociación de gramíneas y leguminosas para proporcionarle al ganado un alimento balanceado (en el caso de las vacas, para que produzcan más leche). Al asociar diferentes especies de pastos, se produce más forraje verde que sembrando Toledo solamente, por tanto, al producir más forraje podremos criar más animales (Balabarca, 2012, p.4).

Por ejemplo, si una vaca es alimentada solo con pasto, hay riesgo que se timpanice y muera, si es alimentada sólo con gramíneas son flacas, pero si la alimentamos con gramíneas y leguminosas (pastos asociados), tendremos una vaca sana y con buena producción de leche.

\section{¿Por qué es importante asociar pastos?}

Balabarca (2012), afirma que el manejo de pastos significa realizar una serie de actividades con la finalidad de obtener buen rendimiento y duración de nuestros pastos, así mismo optimizar su uso. Las principales actividades de manejo de pastos son las siguientes:

Los elementos principales que limitan el establecimiento y mantenimiento de las especies forrajeras en el trópico son: Nitrógeno, Fósforo y Potasio (N, $\mathrm{P}, \mathrm{K})$ y también Calcio, Magnesio y Azufre ( $\mathrm{Ca}, \mathrm{Mg}$ y S). Es muy importante hacer un análisis del suelo antes de iniciar un establecimiento de una pastura para saber sus condiciones físicas, químicas y para poder hacer las correcciones y ajustes necesarios, según los requerimientos de las especies a sembrar.

Los fertilizantes tienen efecto residual y están relacionados con la solubilidad de los mismos, siendo determinantes en la eficiencia del uso que la planta hace de ellos.

\section{Aporte de las leguminosas en asocio con gramíneas}

Incremento de la calidad del forraje: Las leguminosas incrementan el valor nutritivo de la gramínea asociada, particularmente, en lo que se refiere a los contenidos de proteína total y de minerales, a fin de mantener su calidad a través del tiempo, durante la época seca, cuando más las consumen los animales (Balabarca, 2012, p.8).

Aumento en la producción de biomasa vegetal: Las leguminosas incrementan la producción de materia seca en las praderas, cuando éstas se asocian con gramíneas.

- Las leguminosas mejoran la fertilidad del suelo.

- Las leguminosas favorecen mayor rendimiento de forraje e incrementan la carga animal por unidad de superficie cuando se asocian con gramíneas.

- Las leguminosas muestran desventaja para competir con las gramíneas por nutrimentos en pradera asociada.

\section{Materiales y métodos}

El estudio se realizó en la comunidad Yaoya del municipio de Siuna, vía Rosita, ubicada entre las coordenadas $\mathrm{X}=0746477 \mathrm{Y}=1515237$, a una altura de150 metros sobre el nivel del mar de longitud oeste. Es de tipo cuasi experimental, pre test - post test, longitudinal y prospectivo, en una manzana cubierta de pasto Toledo, la que se dividió en dos partes iguales para el desarrollo del estudio. La primera fase consistió en determinar las propiedades físicas sin Arachis y en la segunda, se aplicaron mediciones a los seis meses de establecido este pasto. Se utilizó la técnica observacional para comparar los cambios en las características físicos y químicos en las parcelas y valorar los costos del establecimiento de las mismas. Las variables evaluadas: propiedades físico - químicas del suelo y los costos de establecimiento.

\section{Resultados y discusión}

\section{Resultados de las características físicas del suelo}

En la Tabla 1. Se reflejan los valores que se obtuvieron como resultado de los análisis físicos en ambas parcelas, con Arachis pintoi y sin este. 
Tabla 1. Resultados del análisis físico del suelo.

\begin{tabular}{|c|c|c|c|c|}
\hline \multicolumn{5}{|c|}{ Resultado de análisis físico en pasto sin (Arachis pintoi) } \\
\hline A & $\mathbf{L}$ & $\operatorname{Ar}$ & Textura & Densidad aparente \\
\hline 12.84 & 27.64 & 59.5 & Franco arenoso & 1.30 \\
\hline \multicolumn{5}{|c|}{ Resultado de análisis físico en pasto con (Arachis pintoi) } \\
\hline \multicolumn{3}{|c|}{ Textura boyucos } & \multirow{2}{*}{ Textura } & \multirow{2}{*}{ Densidad aparente } \\
\hline A & L & $\mathrm{Ar}$ & & \\
\hline 9.78 & 30.76 & 59.68 & F.A & 1.33 \\
\hline
\end{tabular}

Partículas del suelo: $\mathrm{A}=$ arena $\quad \mathrm{L}=$ Limo $\mathrm{Ar}=$ Arcilla $\quad$ F.A= Franco Arenoso

\section{Densidad aparente}

Como puede observarse en la Tabla 1. Los valores físicos tuvieron cierta variación, sin embargo esta no se considera significativa.

Con relación al aumento en la densidad aparente, esta contribuye a mejorar los procesos de aireación, infiltración y retención de humedad en el suelo, igualmente a un incremento en la proporción de nutrientes, teniendo en cuenta que para el cálculo del peso de suelo por hectárea, como la conversión de miliequivalente (meq) /1oogramos y partes por millón (ppm) a kilogramos por hectárea, requieren del conocimiento exacto de la densidad aparente, en el caso del suelo que se estudió el contenido de nutrientes, este presentó cambios que se relacionan con la densidad aparente, como se refleja en la tabla anterior.

\section{Cobertura del (Arachis pintoi)}

Durante el estudio, el (Arachis pintoi) cubre el terreno en un 30\%, a los 30 días de establecido. Entre los 60 y los 90 días la cobertura incrementa al 50\% que se mantuvo estable hasta el inicio de la época de verano que limitó el desarrollo del mismo.

Una de las consideraciones importantes a tener en cuenta en un plan de mejoramiento de suelos, a través del uso de coberturas, es la capacidad y la velocidad de colonización en el suelo de la especie que se va a utilizar como cobertura, porque uno de los beneficios de las coberturas en el mejoramiento de suelos, es que con estas se logra reducir la invasión de especies arvenses y se protege el suelo de la erosión y la pérdida de nutrientes por lixiviación.

\section{Resultado de las características químicas}

Tabla 2. Resultados del Análisis químico de suelos tomados antes de sembrar (Arachis pintoi)

\begin{tabular}{|c|c|c|c|c|c|c|c|c|c|c|c|c|c|}
\hline pH & M0 & \multicolumn{3}{|c|}{ Cationes meq/100g } & \multicolumn{7}{c|}{ Cationes (ppm) } \\
\hline & $\%$ & $\mathrm{Al}$ & $\mathrm{Ca}$ & $\mathrm{Mg}$ & $\mathrm{K}$ & $\mathrm{N}$ & $\mathrm{P}$ & $\mathrm{Cu}$ & $\mathrm{Fe}$ & $\mathrm{Mn}$ & $\mathrm{Zn}$ & $\mathrm{B}$ & $\mathrm{S}$ \\
\hline 5.3 & 2.2 & 1.45 & 4.22 & 1.34 & 0.34 & 0.15 & 4.4 & 6.1 & 129.7 & 67.9 & 1.7 & 0.2 & 5.6 \\
\hline
\end{tabular}

$\mathrm{pH}=$ Potencial de Hidrógeno. M.O = Materia orgánica meq = mili equivalente 
Tabla 3. Resultados del análisis químico del suelo seis meses después de establecido el (Arachis pintoi) en la parcela pasto combinado con (Arachis pintoi).

\begin{tabular}{|l|l|l|l|l|l|l|l|l|l|l|l|l|l|}
\hline pH & Mo & \multicolumn{3}{|c|}{ Cationes meq/100g } & \multicolumn{1}{c|}{ Cationes (ppm) } \\
\hline & $\%$ & Al & Ca & Mg & K & N & P & Cu & Fe & Mn & Zn & B & S \\
\hline 5.3 & 2.7 & 1.58 & 4.10 & 1.34 & 0.45 & 0.18 & 4.4 & 6.1 & 223.7 & 68.80 & 1.75 & 0.2 & 5.1 \\
\hline
\end{tabular}

Tabla 4. Resultados del análisis químico del suelo seis meses después de establecido el (Arachis pintoi), en la parcela Pasto sin (Arachis pintoi).

\begin{tabular}{|c|c|c|c|c|c|c|c|c|c|c|c|c|c|}
\hline Ph & Mo & \multicolumn{3}{|c|}{ Cationes meg/100g } & \multicolumn{7}{c|}{ Cationes (ppm) } \\
\hline & $\%$ & $\mathrm{Al}$ & $\mathrm{Ca}$ & $\mathrm{Mg}$ & $\mathrm{K}$ & $\mathrm{N}$ & $\mathrm{P}$ & $\mathrm{Cu}$ & $\mathrm{Fe}$ & $\mathrm{Mn}$ & $\mathrm{Zn}$ & $\mathrm{B}$ & $\mathrm{S}$ \\
\hline 5.3 & 2.2 & 1.6 & 3.6 & 1.34 & 0.35 & 0.15 & 4.4 & 6.1 & 220 & 67 & 1.77 & 0.2 & 5 \\
\hline
\end{tabular}

Tabla 5. Cuadro comparativo de los resultados encontrados en la parcela de pasto con (Arachis pintoi) y pastos $\sin$ (Arachis pintoi)

\begin{tabular}{|c|c|c|c|}
\hline Elementos & $\begin{array}{c}\text { Resultados } \\
\text { general de la parcela }\end{array}$ & $\begin{array}{l}\text { Ganancias y pérdidas de } \\
\text { nutrientes en la parcela pasto con A. pintoi }\end{array}$ & $\begin{array}{c}\text { Ganancias y pérdidas } \\
\text { de nutrientes en la parcela pasto sin } \\
\text { A. pintoi }\end{array}$ \\
\hline $\mathrm{pH}$ & 5,3 & 0 & 0 \\
\hline MO & 2,2 & 0.5 & 0 \\
\hline Al & 1.45 & 0.13 & 0.15 \\
\hline $\mathrm{Ca}$ & 4,22 & -0.12 & -0.62 \\
\hline $\mathrm{Mg}$ & 1,34 & 0 & 0 \\
\hline K & 0,34 & 0.11 & 0.01 \\
\hline $\mathrm{N}$ & 0,15 & 0.03 & 0 \\
\hline$P$ & 4,4 & 0 & 0 \\
\hline $\mathrm{Cu}$ & 6,1 & 0 & 0 \\
\hline $\mathrm{Fe}$ & 129,8 & 93.9 & 90.2 \\
\hline $\mathrm{Mn}$ & 67,9 & 0.9 & -0.9 \\
\hline $\mathrm{Zn}$ & 1,7 & 0.05 & 0.07 \\
\hline$S$ & 5,6 & -0.5 & -0.6 \\
\hline
\end{tabular}


La Tabla 5. Refleja el comportamiento de los nutrientes sobre las características químicas del suelo en pasturas con (Arachis pintoi) y sin este.

Los suelos estudiados tienen como característica principal, la presencia de altos contenidos de sesquióxidos de $\mathrm{Fe}$ y $\mathrm{Mn}$, comunes en suelos tropicales, de color pardo rojizo, con contenidos de bases de $\mathrm{Ca}, \mathrm{Mg}, \mathrm{K}$, por debajo de los parámetros establecidos.

De acuerdo con los resultados que se obtuvieron, se deduce que la cantidad disponible de elementos se encuentra en niveles críticos, esa característica se debe a la influencia del $\mathrm{pH}$ sobre la solución, elementos como Nitrógeno, Fósforo, Potasio, Azufre, Calcio y Magnesio están altamente limitados por las condiciones de acidez, a mayor $\mathrm{pH}$, más limitación en la disponibilidad de dichos elementos.

El azufre experimentó pérdidas con relación a su disponibilidad inicial, se explica porque algunos aniones $\mathrm{SO}_{4}$ tienden a desaparecer en suelos ácidos, formando compuestos insolubles, siendo la fracción disponible del Azufre dependiente del $\mathrm{pH}$. De la misma forma, el Calcio fue el otro elemento que tuvo pérdidas

Todo lo contrario sucedió con el Potasio por su incremento en sus niveles de disponibilidad de 33.8 $\mathrm{kg} / \mathrm{ha}$, que reporta el primer análisis a $45.22 \mathrm{Kg} /$ ha, al final. Así mismo el Hierro, Manganeso y Zinc, se ven favorecidos en su solubilidad, debido a esto, el pH no manifestó cambios durante el tiempo en estudio.

\section{Aporte al contenido de materia orgánica}

Una de las cualidades del (Arachis pintoi), es su aporte al contenido de materia orgánica, a través de la producción de biomasa o materia seca, que se vio reflejada con una ganancia del $23 \%$ de aporte al contenido de materia orgánica en el suelo, seis meses después de establecido, factor que contribuye a mantener y/o mejorar la fertilidad de un suelo.

\section{Costo del establecimiento de la parcela de pasto}

Para que se llevara a cabo el estudio, hubo la necesidad de invertir un total de $C \$ 7,377.00$ (siete mil trescientos setenta y siete), donde se invirtieron $C \$$ 5,085 córdobas en la parcela del sistema de pasto combinado con (Arachis pintoi), y un total de $C \$$ 2,295.00 en la parcela de pastos sin (Arachis pintoi).

Los resultados antes descritos, muestran que en la parcela de pasto con (Arachis pintoi), los costos son más elevados que la parcela solo de pasto, debido a que, se incurre en más mano de obra y manejo; no obstante, después de un tiempo vamos a obtener mayores ingresos económicos para la familia, lo que puede compensar el del establecimiento, también aportar a la conservación y productividad.

\section{Conclusiones}

El (Arachis pintoi) produjo cambios en seis meses sobre las características físicas, aumentó el porcentaje de la densidad aparente, mejoró las partículas del suelo y el contenido de materia orgánica.

En las características químicas con (Arachis pintoi), se obtuvo un aporte de $1.44 \mathrm{~kg} / \mathrm{ha}$ de Nitrógeno, el que está directamente relacionado con el contenido de materia orgánica, la que aumentó en $24.5 \%$. Se encontraron elementos químicos con ganancias y pérdidas en su disponibilidad, por los procesos de cobertura y lixiviación respectivamente.

El costo del establecimiento de la parcela de pastura asociado con (Arachis pintoi), es más alto económicamente para un productor, que una parcela de practica tradicional; sin embargo, sus bondades compensarían en un futuro la inversión.

\section{Recomendaciones}

\section{A productores}

Implementar sistemas de pasto asociado con leguminosas, porque las pasturas asociadas con (Arachis pintoi), ayudan a mejorar el estado físico y químico del suelo. Establecer altas densidades de cobertura y mantener un sistema de riego en épocas secas.

En los oxisoles y suelos con algún grado de acidez, se hace necesaria la práctica de encalamiento, con el fin de neutralizar el hidrogeno y el aluminio intercambiable y proporcionar fósforo, calcio y magnesio en estado disponible para las plantas. 


\section{- A las universidades, organismos e instituciones}

Promover la implementación de pasturas en asociación con leguminosas en alianza con organismos e instituciones que se dedican al rubro ganadero.

Fomentar la investigación continua en la temática que se abordó.

\section{- A estudiantes}

Que continúen en la realización de estudios que profundicen la generación de información sobre las propiedades físicas químicas sobre el suelo, además de una base de datos.

\section{Lista referencia}

Barbosa, J. A. (2014). Propiedades Físicas del suelo. Colombia. Recuperado el 3 de marzo de 2017, de http://www.monografias.com/trabajos65/ propiedadessuelo/propiedadessuelo2. shtml

Budhu. (2007). Formación y Propiedades del suelo. Costa Rica: suelos del trópico.

Mendoza, O. A. (2010). Caracterización del estado actual de los suelos del departamento de león, en base a sus características físicas y sistema de producción. León: Tesis Monográfico. Universidad Nacional Autónomo de León.

Pérez, O. F. (2009). Fertilidad y Fertilización de suelo. Siuna. Cobertura y extracción total de nutrientes en un suelo de trópico húmedo en la amazonia peruana. Recuperado el 28 de marzo de 2017, de https:/www.google. com.ni/webhp?ie=utf-8\&oe=utf-8\&clien$\mathrm{t}=$ firefoxb\&gws_rd=cr\&ei=h_wFWcGcOMOAmQHJzKfQAQ\#q=Establecimiento+de+cultivos+de+cobertura+y+extracci\%$\mathrm{C}_{3 \%} \mathrm{~B}_{3} \mathrm{n}+$ total+de+nutrientes+en+un+s uelo + de $+\operatorname{tr} \% C_{3} \%$ B 3 pico $+h \% C_{3} \% B$ Amedo+en+la+amazonia

Rincón, C. A. (1999). Maní forrajero ((Arachis pintoi), la leguminosa para sistemas sostenibles de producción agropecuaria. Recuperado el 23 de marzo de 2017, de www.agronet.gov.co/www/ docs

Silva, L. d. (2008). Monografia.com. Recuperado el 14 de marzo de 2017, de http://www.monografias.com/trabajos6/elsu/elsu.shtml

Tecnologías y prácticas para pequeños productores agrarios (TECA). (17 de abril de 2015). Reducir la vulnerabilidad de los sistemas de producción agrícola. Recuperado el 23 de marzo de 2017, de http://teca.fao.org/es/read/8332 Uso y manejo de leguminosas como cobertura de suelos para

Tecnologías y prácticas para pequeños productores agrarios (TECA). (1 de junio de 2007). Maní forrajero (Arachis pintoi Frapovickas y Gregory) una alternativa para el sostenimiento de la ganadería. Recuperado el 21 de marzo de 2017, de http://teca.fao.org/es/read/4623

Zapata, R. (1 de noviembre de 2006). La acidez del suelo. Recuperado el 23 de marzo de 2017, de http://www.madrimasd.org/blogs/ universo/2006/11/01/49004 